\title{
Laparoscopic 5-mm port site hernia: a rare but preventable complication of laparoscopy
}

\section{B. Ramesh, Shraddha Neminath Kurkuri*, Satwik Basavaraj Metgud}

Department of Gynecological Endoscopy and Infertility, Dr Ramesh Hospital, Bangalore, Karnataka, India

Received: 21 August 2016

Accepted: 21 September 2016

\section{*Correspondence:}

Dr. Shraddha Neminath Kurkuri,

E-mail: snk3061988@gmail.com

Copyright: () the author(s), publisher and licensee Medip Academy. This is an open-access article distributed under the terms of the Creative Commons Attribution Non-Commercial License, which permits unrestricted non-commercial use, distribution, and reproduction in any medium, provided the original work is properly cited.

\begin{abstract}
The port site hernia (PSH) is a rare but potentially dangerous complication of laparoscopic surgery ranging from early small omental herniation to delayed hernia formation with or without bowel entrapment. Though $5 \mathrm{~mm}$ PSH is a rarer entity compared to $10 \mathrm{~mm} \mathrm{PSH}$, it is a completely preventable cause of morbidity. The aim of this article is to emphasize the importance of fascial closure of trocar site including peritoneum in order to significantly reduce postoperative morbidity as well as to know the importance of early diagnosis to avoid serious subsequent events, in all $10 \mathrm{~mm}$ or larger ports and a few exceptional cases of $5 \mathrm{~mm}$ ports also.
\end{abstract}

Keywords: Complication, Hernia, Laparoscopy, Port site

\section{INTRODUCTION}

The port site hernia (PSH) is a rare but potentially dangerous complication of laparoscopic surgery ranging from early small omental herniations to delayed hernia formation with or without bowel entrapment. The prevalence of herniation followed by laparoscopic surgery is reported to be $0.0002 \%-6 \%$. $^{1}$ The incidence of an incisional hernia following an operative laparoscopic procedure increases with trocar size and is extremely unusual in ports less than $10 \mathrm{~mm}$ in diameter, ranging from $0 \%-0.09 \% .^{2}$ In contrast, the true prevalence of such complications may be higher because some patients have no symptoms and do not return to the surgeon for follow up. ${ }^{1}$

Laparoscopy has become a popular tool in gynaecology. Laparoscopic operations offer advantages like early postoperative recovery and applications of this technique is still expanding. Parallel to the development of new technique, it will also expose patients to new complications. ${ }^{3}$ Tonouchi et al recognized the first report in the literature of a port-site hernia by Fear in the context of gynecological surgery. ${ }^{4}$ In addition to pain, PSH can lead to severe complications, including bowel obstruction, strangulation and perforation. ${ }^{4}$ PSH can be asymptomatic, but occasionally emergency surgery may be required due to above mentioned complications. ${ }^{2}$ Nezhat's review reported that the substantial manipulation of the $5-\mathrm{mm}$ operative port sites required for the surgeries resulted in trocar-site hernias. ${ }^{5}$ Herniation through 5-mm port sites has been shown, particularly when drains have been left in situ. ${ }^{5}$ It is an important yet under-recognised complication of laparoscopic surgery. ${ }^{6}$

To prevent hernias, trocar sites $10 \mathrm{~mm}$ or greater in size are recommended to close at the fascial level and $5 \mathrm{~mm}$ trocar sites are usually closed only at the subcutaneous level because of the difficulty of exposing the fascia through the small skin incision and the rarity of herniation through these trocar sites. ${ }^{3,6}$ Royal College of Obstetricians and Gynaecologists guidelines advice closure of $7 \mathrm{~mm}$ or larger lateral ports and $10 \mathrm{~mm}$ or larger midline ports. ${ }^{7}$ 
The aim of this report is to emphasize the importance of fascial closure of trocar site including peritoneum in order to significantly decrease postoperative morbidity as well as to know the importance of early diagnosis to avoid serious subsequent events. ${ }^{8}$ Almost all PSH have been found in sites greater than or equal to $10 \mathrm{~mm}$ with only few cases reported of $5 \mathrm{~mm}$ site herniation. ${ }^{1}$ We here in report a case of the patient who developed late onset incisional hernia at the lateral $5 \mathrm{~mm}$ port insertion site.

\section{CASE REPORT}

A 68 years old (para 2 living 2) malnourished elderly lady presented with swelling at the incision site over the abdomen in the right lower quadrant since 1 year. Over a period of 3 months swelling increased in the size and was associated with pain also. She was also treated for VDRL positivity. She underwent laparoscopic radical hysterectomy almost 2 years back for Stage 1A moderately differentiated invasive squamous cell carcinoma of cervix. Duration of surgery was almost 2 hours 30 minutes; we had created pneumoperitoneum using carbon-dioxide gas (closed technique of port insertion) and had used sharp pyramidal trocar for both primary (1) and secondary (3) ports. Secondary ports (5 $\mathrm{mm}$ ) were closed subcutaneously only without closing peritoneum and overlying fascia unlike primary port. She recovered well post-operatively and was discharged on day 7 without any complications.

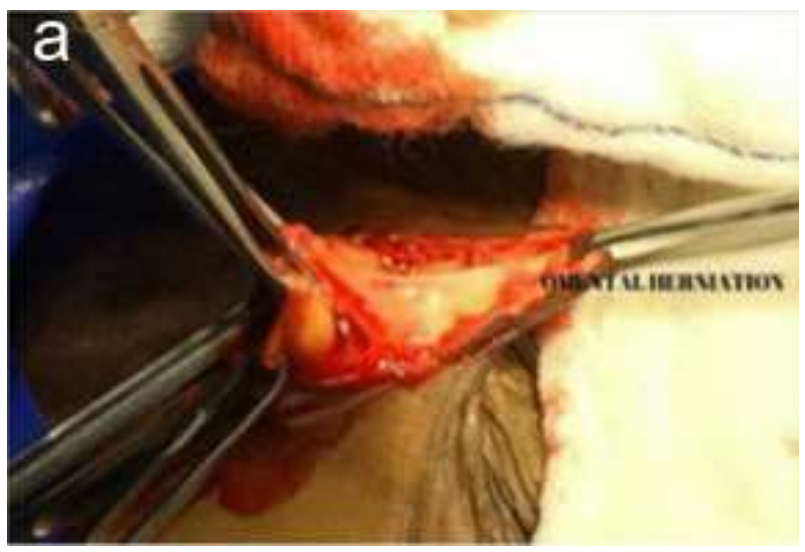

Figure 1a: Laparoscopic $5 \mathrm{~mm}$ port site hernia (right lateral lower port).

Now on examination, Body Mass Index (BMI) was 17 $\mathrm{kg} / \mathrm{m}^{2}$ and per abdominally $2 \times 2 \mathrm{~cm}$ tender swelling was felt at right lower $5 \mathrm{~mm}$ port site. Abdominal ultrasound was done and showed omental herniation through rectus sheath with a defect measuring about $5 \times 10 \mathrm{~mm}$. We did anatomical repair of the $5 \mathrm{~mm}$ port site hernia. Incision was taken over the hernia site along Langerhans line; abdominal wall was opened up. Peritoneum containing the omentum was herniating through the rectus sheath defect (Figure 1a, 1b). Sac was excised after emptying the contents. Peritoneum was closed with Vicryl number 1 and rectus sheath was closed with Prolene number 1 . Subcutaneous tissue and skin were closed with Monocryl number 3-0. She was discharged on the next day without complications. She is doing well now after a month of PSH repair.

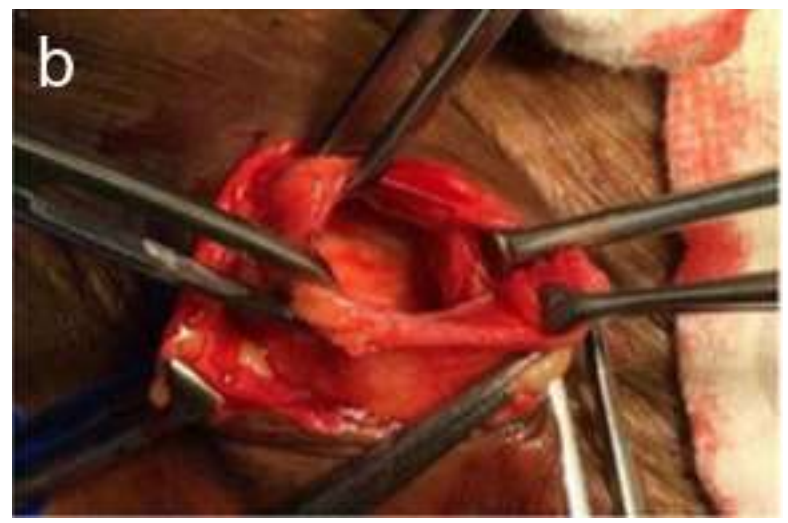

Figure 1b: Peritoneal and rectus sheath defect.

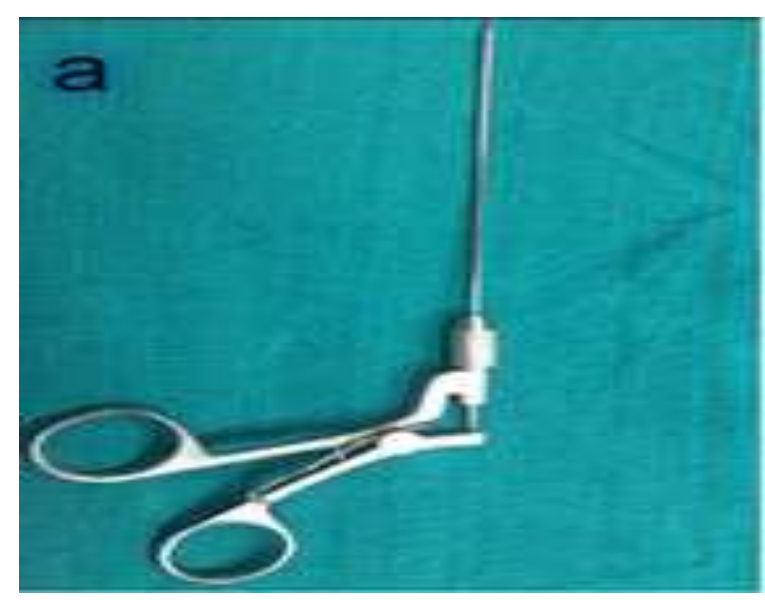

Figure 2a: Port closure needle.

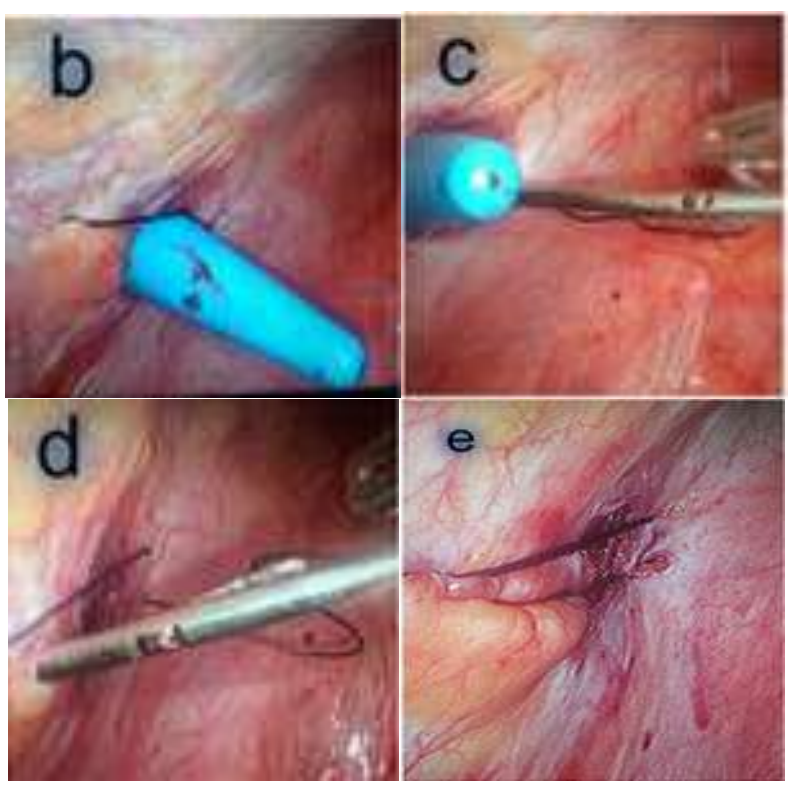

Figure 2b-2e: Port closure technique using port closure needle and port closure cone (suture guide cone). 


\section{DISCUSSION}

Port site hernia is a rare complication following laparoscopic surgery. Laparoscopic surgery has quickly developed, becoming in some cases the gold standard procedure as the preferred mode of access to the peritoneal cavity. Despite the minimal invasiveness, some laparoscopic procedures-related complications can be directly attributed to abdominal access with laparoscopic trocars. The postoperative PSH formation, which is defined as the development of a hernia at the trocar insertion site, is one of them. ${ }^{1}$

Following points regarding PSH have been discussed: first, classification of PSH; second, what might be the factors which had causal relationship to the development of the PSH, what might be the additional option for diagnosis and best management of this disease; lastly, how $5 \mathrm{~mm}$ PSH is preventable.

Tonouchi et al, suggested a classification in which PSH were classified into three types. ${ }^{3}$ The early onset trocar port hernia type was defined as having dehiscence of fascia and peritoneum within 2 weeks, most commonly with small bowel obstruction. When hernia occurs after 2 weeks and has dehiscence of fascial plane with a sac consisting of peritoneum, it is classified as a late onset type port-site hernia. Only a small part of late onset hernias present with intestinal obstruction. The third category includes special types of hernia which have dehiscence of the whole abdominal wall. ${ }^{1}$ We would refer our case to the late onset $5 \mathrm{~mm}$ port-site hernia because there was dehiscence of fascia with a sac consisting of peritoneum with omentum herniating. There are few obvious risk factors for development of lateral $5 \mathrm{~mm}$ port site hernia in our patient. One is malnutrition (BMI: 17 $\mathrm{Kg} / \mathrm{m}^{2}$ ) associated with chronic infection (VDRL positive) and other is the duration of the surgery (about 2 hours 30 minutes) as it was laparoscopic radical hysterectomy for carcinoma cervix, which would definitely hamper the healing process of port site wound. She also had a very thin abdominal wall and through the same port myoma screw was inserted to manipulate the uterus which would also contribute for the occurrence of PSH. Prolonged manipulation may extend or widen the initial defect. $^{5}$

Many factors causing intra-abdominal hernias after laparoscopic surgery have been emphasized. Such as the intra-abdominal pressure overwhelming abdominal wall strength, number of trocars used and their diameters (10 $\mathrm{mm}$ or more $-86 \%$ of all $\mathrm{PSH}$ ), the duration of the procedure (the longer it takes, the higher risk is), the aponeurotic defects in trocar place (incomplete closure of fascia), the insertion technique, the method of withdrawing the trocar, midline trocars, stretching the port site for organ retrieval, the effect of a partial vacuum while port withdrawal, port closure technique, presence of drain at port site, obesity, poor nutrition and operation site infection at wound site. ${ }^{1,3}$
The factors predisposing to PSH can be divided into patient factors and operative factors. The presence of a pre-existing umbilical/paraumbilical hernia has been identified in several reports as a risk factor for PSH. Male gender seemed to be associated with a higher incidence of hernia in one report, without statistical analysis. In another study, the incidence was higher in women on univariate analysis but not in the multivariate analysis. Obesity has been suggested as a predisposing factor in some studies. ${ }^{4}$ Some authors indicated that they believe that because of the substantially thickened preperitoneal space and increased intra-abdominal pressure, there is a tendency to improperly close the fascial defect in obese patients. ${ }^{9}$ The majority of studies did not assess the effect of body mass index (BMI) on the incidence of PSH. One study suggested that there may be a correlation with sudden weight gain and hernia development following surgery rather than obesity itself. ${ }^{9}$ Other patient related factors which increased risk of PSH are diabetes mellitus, chronic obstructive pulmonary disease, arterial insufficiency, immune deficiency, malnutrition, smoking and infection (usually present with late onset type PSH) at incision site. ${ }^{4,9}$

Trocar diameter has been widely reported as a factor in development of PSH. Most of them are at port sites measuring $10 \mathrm{~mm}$ or more. However, there are many other reports in the literature of herniation through $5 \mathrm{~mm}$ ports, particularly in children and indeed a single report of herniation through a $3 \mathrm{~mm}$ port site. Primary port insertion is either by closed or open technique. The closed technique usually involves Veress needle insufflation followed by blind insertion of a trocar. In the open technique (Hasson or similar), the abdominal wall is opened, and the port inserted under direct vision. It has been suggested that in the case of closed insertion, even when the fascia is sutured, the incidence of PSH is higher. Fascial closure can be more difficult when the closed technique has been used, particularly in obese patients. None of the other studies were of a suitable methodology to compare the 2 insertion techniques. Secondary ports are less often the site of hernia development, but hernias do occur at secondary port sites. The type of trocar used is thought to be a determining factor. Broadly, they can be divided into 2 types: cutting trocars and dilating trocars. The most common cutting trocars in use are reusable metal pyramidal trocars and disposable metal blade trocars with or without a sprung protective sleeve. They generally require less force to use but have a higher incidence of complications, such as bleeding, pain, and hernias. Dilating or "radially expanding" trocars bluntly separate abdominal wall tissues. They are thought to be associated with less bleeding and pain, although they require greater application of force to insert which could increase internal organ injury. Newer hybrid designs have also been developed in an effort to minimize these problems. Laparoscopic procedures that require multiple trocars are associated with a higher frequency of hernias. In an experimental study of wounds relative to the type of 
trocar, it was found that a non-bladed trocar created smaller peritoneal port site wounds than a conventional pyramidal tip. Some authors have also reported a lower prevalence of hernias with the use of a paramedian incision and non-bladed trocars which have a conical tip. Nevertheless, port site created by a non-bladed trocar requires fascial closure. There is evidence to support the notion that the site of trocar placement may have a bearing on the subsequent development of $\mathrm{PSH}^{4}$ As Azurin et al and other authors have shown, most PSHs developed in the midline rather than at the lateral site. Plaus and Duron et al stated that because there is overlapping of muscles and 2 fascial layers, the lateral site is less susceptible to dehiscence. ${ }^{9}$

The clinical course of PSH can be variable and depends on the extent and nature of the herniated content. Mild symptoms such as slight nausea and vague abdominal pain, both of which are most frequently seen in the early normal postoperative course after abdominal surgery, could be the first and only complaints at the early stage of this complication. Thus, the diagnosis may be delayed. ${ }^{8}$ Most hernias present within 10 days from the procedures, even though delayed hernias have been reported up to a year from the initial operation. ${ }^{10}$ In case of strangulation, it is typically of a Richter's type, in which omentum rather than bowel is the herniating tissue which presents later and with minimal symptoms. ${ }^{4}$ Swelling and pain at the incision site may be difficult to differentiate from a hematoma or wound infection. ${ }^{10}$ Even such rare entity as postoperative hernia at the previous site of introduction of the smallest laparoscopic trocar in most lateral side of the abdomen can be responsible for acute intestinal obstruction and, therefore, must be kept in mind during diagnostic differentiation because time of surgery is a most crucial thing in the management of incarcerated hernias. ${ }^{1} \mathrm{CT}$ scan is a useful adjunct to differentiate port site hematoma from incarcerated small bowel and ileus from small bowel obstruction, colonic neoplasm and adhesions, acts as a vital guide to intervention. ${ }^{1,10}$ The management of most of these hernias includes access to the hernia by extending the trocar site, laparoscopy or an explorative laparotomy and then reduction of the hernia and further surgeries based on the bowel viability. Boughey et al have reported four cases of Richter's hernia that occurred at a port site after laparoscopic surgery. ${ }^{8}$

Prevention is the key. Finally, there are some measures suggested to prevent trocar port-site hernia. To the best of our knowledge they are mentioned as follows. The use, whenever possible, of trocars of smaller diameter, avoiding extreme manipulation of trocars, introducing trocars with a Z-incision technique, closing fascial defect, direct vision deflation of air and removing of trocars. ${ }^{1}$ Moreover, recent publications have demonstrated that radially expanding type trocars could be useful to avoid the necessity of closing the fascial defect. Many authors have recommended the deflation of pneumo-peritoneum prior to port removal so that omentum and intestines would not be drawn into the fascial defect. The trocar must be removed under direct visualization, and the wound should be digitally explored, assuring that no bowel has been entrapped. Adequate closure of the fascia for wounds larger than $10 \mathrm{~mm}$ is necessary. In general, the closure of the fascia in a wound of $10 \mathrm{~mm}$ or smaller is questioned. However, some surgeons recommend closing $5 \mathrm{~mm}$ port in patients who are older than 60 years of age with a BMI above $25 \mathrm{~kg} / \mathrm{m}^{2}$, whose laparoscopic surgery duration is longer than 90 minutes and after extensive trocar manipulation both of which may also lead to an extension of an initial incision. The peritoneum should be incorporated into the fascial closure to obliterate the preperitoneal space, thereby preventing herniation. ${ }^{1}$ Elective closure of rectus irrespective of port size should be considered in case of prolonged manipulation through port or repeated insertion of a trocar, both of which may lead to an extension of an initial incision. ${ }^{7}$ Some authors mentioned that closure should be done under direct vision, and it should incorporate all layers of the abdominal wall to eliminate the peritoneal defect. Some surgeons recommend the use of a fascial closure device, a spinal cord needle, a suture carrier, a $2 \mathrm{~mm}$ trocar, an Endoclose suture device, or a Deschamps needle to close the fascia and the peritoneum

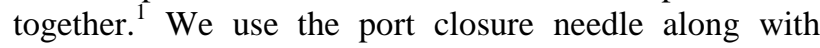
suture guide cone for closure of $10 \mathrm{~mm}$ or larger ports and leave smaller unclosed except in few cases (Figures $2 \mathrm{a}, 2 \mathrm{~b}, 2 \mathrm{c}, 2 \mathrm{~d}, 2 \mathrm{e})$. Elashry et al described a prospective randomized study demonstrating that the CarterThomason device was faster and resulted in fewer portclosure-related complications among eight different techniques tested. Insertion of a SURGICEL plug into the muscular layer of trocar wounds has also been proposed by Chiu et al. Tangential insertion of a trocar through the abdominal wall has also been effective in reducing the size of fascial defects. ${ }^{8}$

Thus, awareness and knowledge of the predisposing factors, early diagnosis, its complications and modification of techniques may help to reduce the risk of PSH.

\section{CONCLUSION}

Hernia at $5 \mathrm{~mm}$ laparoscopic port site is extremely rare, but attention should be paid to a possible occurrence of the hernia. It is a completely preventable cause of morbidity that requires a second surgical procedure to repair. Prompt investigation and further intervention may reduce unfavourable events if a $\mathrm{PSH}$ is suspected. CT scan is a helpful adjunct to diagnose PSH. Every effort should be done to repair the fascial and peritoneal defects to prevent the port site hernia. All fascial defects larger than or equal to $10 \mathrm{~mm}$ should be closed with peritoneum. There is no evidence to recommend routine closure of $5 \mathrm{~mm}$ port incisions except in a few cases, which again depends of the individual decision of the operating surgeon. 
Funding: No funding sources

Conflict of interest: None declared

Ethical approval: Not required

\section{REFERENCES}

1. Dulskas A, Lunevičius R, Stanaitis J. A case report of incisional hernia through a $5 \mathrm{~mm}$ lateral port site following laparoscopic cholecystectomy. J Minim Access Surg. 2011;7(3):187-9.

2. Kilic GS, Bildaci TB, Tapisiz OL, Alanbay I, Walsh T, Swanson O. Trocar site hernia on an 8-mm port following robotic-assisted hysterectomy. Chin Med Assoc. 2014;77(2):112-4.

3. Iino T, Oishi H, Yamane T, Hirai E, Kameoka S, Fukunga M. A Case of Incarcerated Hernia at the $5 \mathrm{~mm}$ Port Site after Removal of Tube Drain. SAGES. 2012.

4. Bunting DM. Port-site hernia following laparoscopic cholecystectomy. JSLS. 2010;14(4):490-7.

5. Yamamoto M, Minikel L, Zaritsky E. Laparoscopic 5-mm trocar site herniation and literature review. JSLS. 2011;15(1):122-6.

6. Owens M, Barry M, Janjua AZ, Winter DC. A systematic review of laparoscopic port site hernias in gastrointestinal surgery. Surgeon. 2011;9(4):218-24.
7. Haider J, AlHamza A, George A, Amelia D, Nitish N. A 5-mm Port-Site Hernia Following a Laparoscopic Myomectomy Resulting in Bowel Obstruction. J Gynecol Surg. 2014;30(4):247-50.

8. Itoh T, Fuji N, Taniguchi H, Watanabe T, Kosuga T, Kashimoto K, Naito K. Port site herniation of the small bowel following laparoscopy-assisted distal gastrectomy: a case report. Journal of Medical Case Reports. 2008;2:48.

9. Agaba EA, Rainville H, Ikedila O, Vemulapali P. Incidence of Port-Site Incisional Hernia After SingleIncision Laparoscopic Surgery. JSLS. 2014;18(2):204-10.

10. Rammohan A, Naidu RM. Laparoscopic port site Richter's hernia- An important lesson learnt. International Journal of Surgery Case Reports. 2011;2:9-11.

Cite this article as: Ramesh B, Kurkuri SN, Metgud SB. Laparoscopic 5-mm port site hernia: a rare but preventable complication of laparoscopy. Int $\mathbf{J}$ Reprod Contracept Obstet Gynecol 2016;5:4056-60. 